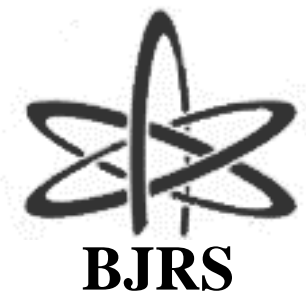

BRAZILIAN JOURNAL

$\mathrm{OF}$

RADIATION SCIENCES

03-1A (2015) 01-22

\title{
Estudo de caso sobre o efeito da radiação cósmica em sistemas embarcados em aeronaves
}

\author{
A. C. M. Prado ${ }^{a, b}$; M. A. Pereira ${ }^{a, b}$; C. A. Federico ${ }^{b}$; O. L. Gonçalez ${ }^{b}$ \\ ${ }^{a}$ Instituto Tecnológico de Aeronáutica, 12228-900, São José dos Campos-São Paulo, Brasil \\ ${ }^{b}$ Divisão de Física Aplicada, Instituto de Estudos Avançados, 12228-001, São José dos Campos-São Paulo, Brasil \\ adriane.acm@hotmail.com
}

\begin{abstract}
RESUMO
Nêutrons de alta energia, gerados a partir da interação da radiação cósmica com átomos da atmosfera, podem provocar efeitos indesejáveis nos dispositivos aviônicos. Estes efeitos são denominados como Single Event Effects (SEE) e podem ocorrer principalmente em computadores de bordo de aeronaves provenientes da mudança do estado lógico de células de memória ou em interrupções funcionais, podendo comprometer a segurança de voo. Os efeitos de SEE devem ser previamente avaliados e inseridos no processo de análise de segurança com a finalidade de determinar a susceptibilidade dos dispositivos a falhas por SEE. A taxa de SEE pode ser avaliada separadamente para nêutrons térmicos e para nêutrons rápidos com energia acima de $10 \mathrm{MeV}$. Este trabalho apresenta um estudo prospectivo da susceptibilidade à radiação para um tipo específico de memória SRAM, nos períodos de máximo e mínimo solar, em situações de voo equatorial e polar na altitude típica de voo das aeronaves atuais, bem como, em altitudes superiores, próximas ao máximo de Pfotzer. Este estudo foi realizado por meio de estimativas dos fluxos de partículas empregando-se os códigos EXPACS e QARM, avaliando a taxa esperada de SEE devida a nêutrons térmicos e a nêutrons rápidos separadamente. A distribuição em energia e a fluência de nêutrons no interior da aeronave são influenciadas pela massa total da aeronave, de forma que também são discutidas as influências da massa da aeronave na taxa de SEE do dispositivo, utilizando-se como casos exemplo, aeronaves de três categorias de peso máximo de decolagem.
\end{abstract}

Palavras-chave: radiação cósmica, aviônicos, falhas.

\section{INTRODUCÃO}


O ambiente de voo de aeronaves é sujeito a radiações ionizantes presentes na atmosfera, produzidas a partir de interações da radiação cósmica primária, oriunda do espaço, com os átomos da atmosfera. Estas interações criam chuveiros secundários de radiação formados principalmente na troposfera e baixa estratosfera. Em altitudes típicas de voo a componente neutrônica possui maior influência tanto para o equivalente de dose ambiente, quanto para os efeitos transientes (Single Event Effect - SEE) que ocorrem em dispositivos eletrônicos (FEDERICO, 2011). O fluxo destas radiações é modulado principalmente pelo ciclo solar e pelo clima espacial, aumentando com a latitude geomagnética e com a altitude, atingindo um máximo entre 15 e 20 km, denominado máximo de Pfotzer. O período de máximo e mínimo da atividade solar é anticorrelacionado com a taxa de contagem de nêutrons no solo (FEDERICO, 2011).

Quando ocorre uma ejeção de massa coronal do Sol, o fluxo de partículas e a taxa de dose também são alterados na atmosfera, e estes eventos podem provocar mudanças significativas no fluxo de radiação em altitudes típicas de voo e consequentemente na taxa de efeitos da radiação conhecidos como "Single Event Effects" (SEE), que serão explicados em maiores detalhes mais adiante (DYER, 2003).

A radiação atmosférica também é alterada devido à latitude geomagnética sendo a intensidade de raios cósmicos mínima no equador magnético, enquanto que nos polos ela apresenta seu maior valor. A maior intensidade nas regiões polares ocorre pelo fato de o campo magnético terrestre não apresentar influência sobre as partículas que incidem verticalmente sobre essas regiões (MENDONÇA, 2011). Mais precisamente podemos dizer que, para maiores latitudes geomagnéticas (nos polos) a rigidez de corte do campo magnético terrestre é menor. A rigidez de corte do campo magnético terrestre é a grandeza física que descreve a capacidade ou não que uma partícula carregada tem de atravessar, ficar aprisionada, ou ser repelida pelas linhas do campo magnético da Terra. Assim, quanto menor for a rigidez de corte do campo magnético, mais facilmente as partículas conseguem atravessa-lo e penetrar na atmosfera terrestre.

A variação do fluxo de nêutrons atmosféricos aumenta significativamente com a altitude (INTERNATIONAL ELECTROTECHNICAL COMMISSION, 2006) até atingir um máximo em torno de 15 a $20 \mathrm{~km}$ conforme pode ser observado na Figura 1 à esquerda. Como a intensidade dos raios cósmicos é alterada em função da latitude geomagnética o mesmo ocorre com o fluxo de nêutrons, como apresentado na Figura 1 à direita. 
Os resultados apresentados na Figura 1 foram obtidos por meio do código EXPACS (SATO, 2006; SATO, 2008). A variação do fluxo integral de nêutrons em função da altitude foi obtida para a latitude de $45^{\circ}$ com o potencial de desaceleração solar de 465 MV. Já a variação do mesmo fluxo integrado de nêutrons em função da latitude foi obtida para 39000 e $40000 \mathrm{ft}$, considerando o mesmo valor de potencial de desaceleração solar.

Figura 1: Variação do fluxo de nêutrons atmosféricos com a altitude (esquerda) e com a latitude (direita) calculados com o código EXPACS

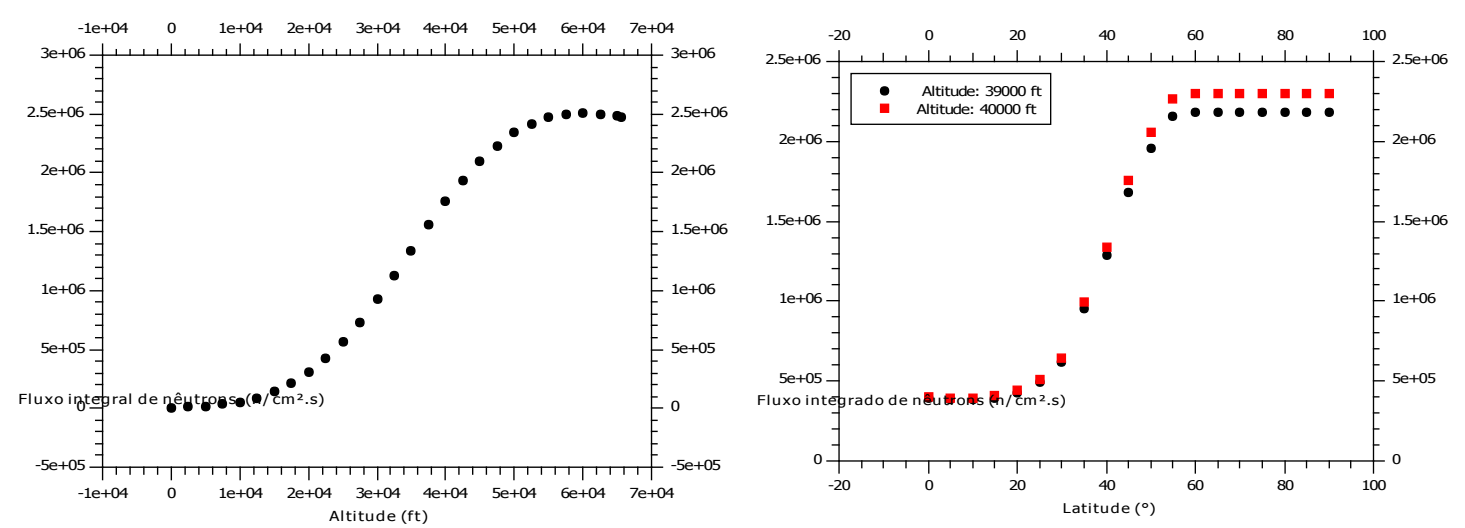

Os dispositivos aviônicos estão inseridos neste ambiente crítico durante o voo e são susceptíveis aos efeitos da radiação conhecidos como "Single Event Effects" (SEE) produzidos principalmente por nêutrons. Devido ao desenvolvimento de aeronaves com o teto operacional mais alto, maior autonomia e o aumento no fluxo aéreo, estudos dos efeitos da radiação em dispositivos aviônicos têm se tornado importantes no que diz respeito à segurança de voo, uma vez que estes efeitos podem causar falhas em dispositivos críticos. Com a crescente miniaturização e alto grau de integração dos componentes eletrônicos, indo da escala de micro para nanometros, têm-se observado um aumento significativo na susceptibilidade destes componentes aos efeitos da radiação ionizante. Todos os dispositivos aviônicos estão sujeitos a falhas induzidas pela radiação e as mais críticas são aquelas que ocorrem principalmente nos computadores de bordo de aeronaves provenientes da mudança do estado lógico de células de memória (Single Event Upsets ou Multiple Bit Upsets) ou de interrupções funcionais (Single Event Fault Interrupt), podendo comprometer a segurança de voo. 
Os SEEs já foram identificados como a causa mais provável de algumas avarias em um Airbus A330 - 303 operado pela Qantas Airways no ano de 2008 resultantes de comandos indevidos no piloto automático da aeronave por falha no computador de bordo. De acordo com o relatório emitido pela Australian Transport Safety Bureau (ATSB, 2011) a unidade inercial da aeronave enviou dados errôneos ao sistema de controle de voo ocasionando uma mudança brusca de atitude que lançou os passageiros ao teto da aeronave. Cerca de 110 dos 303 passageiros e 9 dos 12 membros da tripulação ficaram feridos. A Figura 2 apresenta fotos deste incidente.

Figura 2: Fotos do incidente com a aeronave Airbus A330 - 303

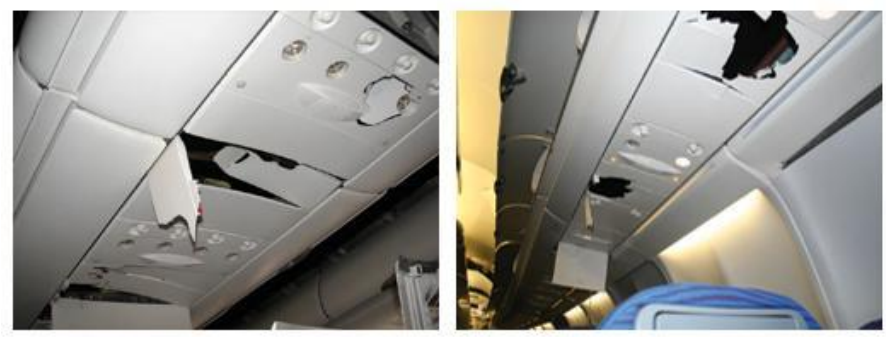

Fonte: Extraído de (COOPER, 2012).

Os efeitos da radiação em circuitos eletrônicos podem aparecer como uma mudança permanente ou transitória dos parâmetros elétricos dos componentes do circuito, seu mau funcionamento ou, até mesmo, sua falha completa e inoperacionalidade. Estes efeitos podem ser classificados como estocásticos ou cumulativos, podendo, também ser reversíveis ou irreversíveis (GONÇALEZ, 2012).

Os efeitos cumulativos dependem da dose total acumulada ao longo do tempo de exposição do componente à radiação e podem ser divididos em Total Ionizing Dose (TID) causado pelo aprisionamento das cargas liberadas pela radiação e nos efeitos de Displacement Damage (DD) causado pelo deslocamento de átomos da rede cristalina pela colisão elástica ou inelástica de uma partícula pesada incidente.

Os SEE são efeitos estocásticos (probabilísticos) e podem ser divididos em efeitos permanentes, caracterizados por causar danos irrecuperáveis aos componentes eletrônicos, e efeitos transientes, geralmente associados à coleta rápida de cargas elétricas liberadas em uma região sensível do dispositivo pela passagem de uma partícula fortemente ionizante. 
O nêutron não possui carga elétrica, mas pode causar SEEs na eletrônica por nêutrons rápidos através de reações nucleares produzindo fragmentos ionizantes, fragmentos de fissão ou núcleos de recuo que ionizam o meio. E também pode causar efeitos na eletrônica por meio de nêutrons térmicos através de reações com contaminantes como ${ }^{10} \mathrm{~B}$ e ${ }^{235} \mathrm{U}$ presentes na microeletrônica que se fissionam na absorção dessas partículas. Como exemplo é apresentada na equação 1, a seguir, a fissão do isótopo de massa atômica 10 do Boro.

$$
\mathrm{n}+{ }^{10} \mathrm{~B} \longrightarrow{ }^{4} \mathrm{He}+{ }^{7} \mathrm{Li}
$$

De acordo com recomendações técnicas da IEC (INTERNATIONAL ELECTROTECHNICAL COMMISSION, 2006), estes efeitos devem ser previamente avaliados e inseridos no processo de análise de segurança com a finalidade de determinar a susceptibilidade a falhas dos dispositivos por SEE. Uma metodologia sugerida pela IEC é avaliar taxa de SEE separadamente para nêutrons térmicos e nêutrons rápidos com energia acima de $10 \mathrm{MeV}$.

O objetivo deste trabalho é apresentar um estudo prospectivo da susceptibilidade à radiação cósmica para um tipo específico de memória SRAM, nos períodos de máximo e mínimo solar, em situações de voo equatorial e polar na altitude típica de voo das aeronaves atuais, bem como, em altitudes superiores, próximas ao máximo de Pfotzer, conforme tendência observada nos projetos de aeronaves futuras. Este estudo foi realizado por meio de estimativas de fluxos de partículas empregando-se os códigos EXPACS (SATO, 2006; SATO, 2008) e QARM (LEI, 2004; LEI, 2006). Com base nestes resultados é calculada a taxa esperada de SEE devida a nêutrons térmicos e nêutrons rápidos. A distribuição em energia e a fluência de nêutrons no interior da aeronave são influenciadas pela massa total da aeronave, de forma que também são discutidas as influências da massa da aeronave na taxa de SEE do dispositivo, utilizando-se como caso exemplo, aeronaves de três categorias de peso máximo de decolagem.

\section{MATERIAIS E MÉTODOS}


Uma metodologia sugerida pela IEC para avaliar a susceptibilidade de dispositivos eletrônicos embarcados é determinar a taxa de SEE esperada do dispositivo quando operado sob radiação. A taxa esperada de SEE do dispositivo depende da seção de choque do mesmo, que pode ser obtida em bibliotecas de dados oriundos de testes realizados previamente em dispositivos semelhantes ou ser obtida através de ensaios do dispositivo de interesse em um campo de radiação conhecido. Assim, pode-se dizer que a susceptibilidade de um dispositivo aos efeitos de SEE é quotada por meio da sua "Seção de choque SEE". A seção de choque SEE do dispositivo é proporcional à área sensível do mesmo entre outros fatores, e expressa o número de falhas por unidade de fluência de radiação incidente. Dessa maneira, para se prever a quantidade de falhas num determinado tempo de voo basta multiplicar a secção de choque do tipo de SEE de interesse pela fluência total de radiação prevista para o voo.

A taxa total SEE $\left(\mathrm{R}_{\text {total }}\right)$ esperada é apresentada na equação 2.

$$
R_{\text {Total }}=R_{\text {rápidos }}+R_{\text {térmicos }}
$$

As equações 3 e 4 apresentam a taxa de SEE para nêutrons rápidos $\left(R_{\text {rápidos }}\right)$ e térmicos $\left(R_{\text {térmicos }}\right)$, respectivamente.

$$
\begin{gathered}
R_{\text {rápido }}=\sigma_{\text {rápidos }} \cdot \phi_{\text {rápidos }} . \\
R_{\text {térmico }}=\sigma_{\text {térmicos }} \cdot \phi_{\text {térmicos }} .
\end{gathered}
$$

Onde $\sigma_{\mathrm{i}}$ é a seção de choque do dispositivo e $\phi_{i}$ é o fluxo de nêutrons a qual o dispositivo é exposto durante a sua utilização real ( $\mathrm{i}=$ térmicos, rápidos).

Os valores das seções de choque para nêutrons térmicos e rápidos de duas memórias SRAMs típicas utilizadas em sistemas aviônicos é apresentada na tabela 1, cujos dados foram extraídos da IEC (2008). O fluxo de nêutrons ao qual o dispositivo é exposto durante sua utilização pode ser determinado pelos códigos QARM e EXPACS. 
Tabela 1: Dados de seção de choque para nêutrons rápidos e térmicos de duas SRAMs típicas de uso em aviônicos.

\begin{tabular}{ccccc}
\hline Dispositivo & Fabricante & $\sigma_{\text {rápidos }}\left(\mathrm{cm}^{2} / \mathrm{bit}\right)$ & $\sigma_{\text {térmico }}\left(\mathrm{cm}^{2} / \mathrm{bit}\right)$ & $\sigma_{\text {térmico }} / \sigma_{\text {rápido }}$ \\
\hline $\begin{array}{c}\text { D1: } \\
\text { M5M5408AFP }\end{array}$ & Mitsubishi & $1,88 \times 10^{-13}$ & $1,8 \times 10^{-13}$ & 0,96 \\
\hline $\begin{array}{c}\text { D2: } \\
\text { MCM69P737 }\end{array}$ & Motorola & $6,66 \times 10^{-15}$ & $1,62 \times 10^{-14}$ & 2,4 \\
\hline
\end{tabular}

O código QARM fornece o espectro de nêutrons para diferentes altitudes para data e local especificado enquanto o código EXPACS fornece o espectro de partículas para diferentes altitudes, diferentes valores de rigidez de corte vertical (a qual determina o local de interesse de simulação do espectro atmosférico), para diferentes valores de potencial de desaceleração solar e, em particular para o espectro de nêutrons, leva em consideração a influência da massa da aeronave. Os dados de input para o QARM e EXPACS são apresentados nas tabelas 2 e 3 respectivamente.

Tabela 2: Dados de input do QARM.

\begin{tabular}{|c|c|c|c|c|}
\hline Parâmetros & Altitude & Data & Local & $\mathrm{Kp}$ \\
\hline Mínimos & $11000 \mathrm{~m}$ & $\begin{array}{c}\text { 22/11/1986 } \\
\text { (mínimo solar) }\end{array}$ & $\begin{array}{c}\text { Latitude: } 90^{\circ} \\
\text { Longitude: } 0^{\circ} \\
\text { (baixo valor de rigidez de cor- } \\
\text { te, polos geográficos) }\end{array}$ & 0 \\
\hline Máximos & $18000 \mathrm{~m}$ & $\begin{array}{c}\text { 16/07/1991 } \\
\text { (máximo solar) }\end{array}$ & $\begin{array}{l}\text { Latitude: } 10^{\circ} \\
\text { Longitude: } 85^{\circ} \\
\text { (alto valor de rigidez de corte, } \\
\text { região equatorial) }\end{array}$ & 0 \\
\hline
\end{tabular}

As datas escolhidas representam o período de mínimo e máximo solar, os valores médios mensais foram obtidos da FAA e são apresentados na Figura 3 (FEDERICO, 2011). 
Figura 3: Potencial solar mensal médio no período de janeiro de 1958 a março de 2011

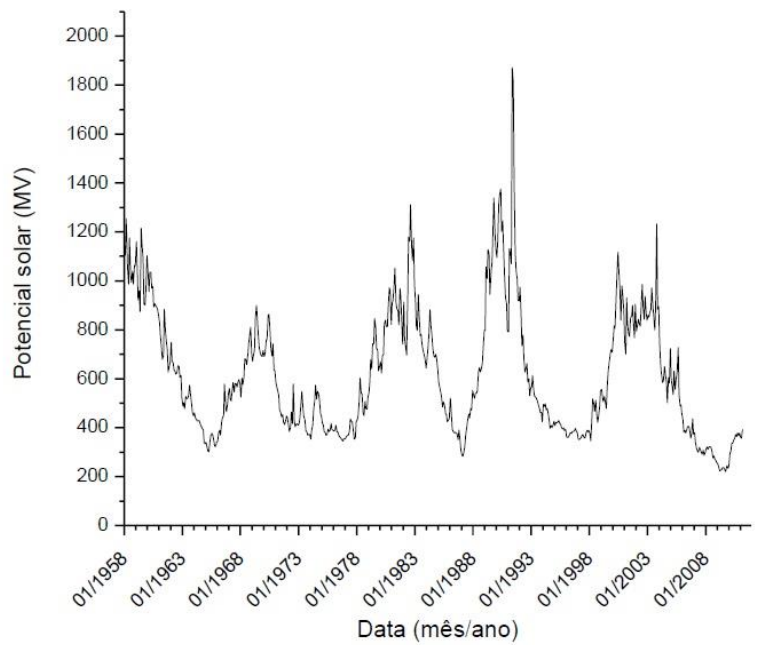

Fonte: Extraído de (FEDERICO, 2011)

Tabela 3. Dados de input do EXPACS

\begin{tabular}{|c|c|c|c|c|}
\hline Parâmetros & Altitude & $\begin{array}{l}\text { Potencial de desacelera- } \\
\text { ção solar }\end{array}$ & Rigidez de corte vertical & $\mathrm{LEP}^{1}$ \\
\hline Mínimos & $11000 \mathrm{~m}$ & 465 MV (mínimo solar) & $\begin{array}{c}0 \mathrm{GV} \\
\text { (localização: polos geo- } \\
\text { gráficos) }\end{array}$ & $\begin{array}{c}0 \\
0,08\end{array}$ \\
\hline Máximos & $18000 \mathrm{~m}$ & 1700 MV (máximo solar) & $\begin{array}{c}17 \mathrm{GV} \\
\text { (localização: região equa- } \\
\text { torial) }\end{array}$ & $\begin{array}{c}0,8 \\
4\end{array}$ \\
\hline
\end{tabular}

Local Effect Parameter (LEP): Parâmetro de efeito local, que representa a massa total da aeronave, dada em centenas de toneladas.

O código QARM não considera o fator de massa da aeronave e corresponde, portanto, ao espaço livre na localização da aeronave (fluxo incidente na aeronave). Os dados obtidos com o EXPACS correspondem à posição do piloto.

\section{RESULTADOS E DISCUSSÕES}

As tabelas 4 e 5 apresentam as diferenças percentuais entre os resultados obtidos com o código QARM e os obtidos através do código EXPACS para a faixa de energia de nêutrons térmicos 
$(<0,25 \mathrm{eV})$ e para a faixa de energia de nêutrons rápidos com E $>10 \mathrm{MeV}$, tomando-se como referência o resultado do código QARM.

Tabela 4: Diferença percentual entre o fluxo de nêutrons no espaço livre obtidos por meio do QARM e os obtidos com o EXPACS para $11 \mathrm{~km}$ de altitude.

\begin{tabular}{ccccccc}
\hline & \multicolumn{2}{c}{ FLUXO RÁPIDO $\left(\mathrm{n} / \mathrm{cm}^{2} . \mathrm{s}\right)$} & \multicolumn{2}{c}{ FLUXO TÉRMICO $\left(\mathrm{n} / \mathrm{cm}^{2} . \mathrm{s}\right)$} \\
\hline $11000 \mathrm{~m}$ & QARM & EXPACS & $\begin{array}{c}\text { DIFERENÇA } \\
\%\end{array}$ & QARM & EXPACS & $\begin{array}{c}\text { DIFERENÇA } \\
\%\end{array}$ \\
\hline $0 \mathrm{GV} 465 \mathrm{MV}$ & 1,29 & 1,364 & $-5,74$ & 0,19 & 0,237 & $-23,2$ \\
\hline 0 GV 1700 MV & 0,89 & 0,597 & 32,5 & 0,13 & 0,104 & 20,7 \\
\hline 17 GV 465 MV & 0,19 & 0,192 & $-2,31$ & 0,03 & 0,034 & $-23,0$ \\
\hline $\begin{array}{c}17 \text { GV 1700 } \\
\text { MV }\end{array}$ & 0,18 & 0,145 & 17,1 & 0,03 & 0,026 & 0,35 \\
\hline
\end{tabular}

Tabela 5: Diferença percentual entre o fluxo de nêutrons no espaço livre obtidos por meio do QARM e os obtidos com o EXPACS para $18 \mathrm{~km}$ de altitude.

\begin{tabular}{|c|c|c|c|c|c|c|}
\hline & \multicolumn{2}{|c|}{ FLUXO RÁPIDO $\left(\mathrm{n} / \mathrm{cm}^{2} . \mathrm{s}\right)$} & \multicolumn{3}{c|}{ FLUXO TÉRMICO (n/cm $\left.{ }^{2} . \mathrm{s}\right)$} \\
\hline $18000 \mathrm{~m}$ & QARM & EXPACS & $\begin{array}{c}\text { DIFERENÇA } \\
\%\end{array}$ & QARM & EXPACS & $\begin{array}{c}\text { DIFERENÇA } \\
\%\end{array}$ \\
\hline $0 \mathrm{GV} 465 \mathrm{MV}$ & 2,59 & 2,61 & $-0,9$ & 0,37 & 0,47 & $-27,0$ \\
\hline $0 \mathrm{GV} 1700 \mathrm{MV}$ & 1,65 & 0,95 & 42,1 & 0,23 & 0,17 & 25,9 \\
\hline $17 \mathrm{GV} 465 \mathrm{MV}$ & 0,25 & 0,24 & 5,80 & 0,04 & 0,04 & $-17,1$ \\
\hline $17 \mathrm{GV} 1700 \mathrm{MV}$ & 0,23 & 0,18 & 24, & 0,03 & 0,03 & 6,12 \\
\hline
\end{tabular}

Os maiores valores de fluxo, tanto rápido quanto térmico, para ambas altitudes ocorrem durante o período de mínimo solar e voos de rotas polares (465 MV e $0 \mathrm{GV}$ ), demonstrando a previsão teórica de que o fluxo de nêutrons aumenta para maiores latitudes como na região polar (latitude de $90^{\circ}$ ) e durante o período de mínimo solar. Neste período o fluxo de nêutrons é maior, pois as partículas que chegam à Terra são de origem galáctica e possuem maiores energias quando comparadas com partículas oriundas do sol. Por outro lado, quando o sol está em seu máximo (Potencial de desaceleração solar igual a $1700 \mathrm{MV}$ ) cria-se um escudo de partículas solares que bombardeiam a Terra e impedem que partículas vindas da galáxia cheguem com maior 
freqüência na atmosfera terrestre. Como as partículas do sol são de menores energias que as partículas da radiação cósmica galáctica, conseqüentemente, o fluxo de nêutrons na atmosfera é menor. Nota-se que este decréscimo ocorre tanto em rotas polares $(0 \mathrm{GV})$ quanto em rotas equatoriais $(17 \mathrm{GV})$.

A Figura 4 apresenta a variação do fluxo de nêutrons rápidos acima de $10 \mathrm{MeV}$ (esquerda) e de nêutrons térmicos (direita) calculado pelo código EXPACS para altitude de $11000 \mathrm{~m}$ em função da massa da aeronave, nas situações de máximo e mínimo solar para rotas polares e equatoriais.

Figura 4: Variação do fluxo de nêutrons rápidos e fluxo de nêutrons térmicos na posição do piloto em função da massa da aeronave para $11 \mathrm{~km}$ de altitude.

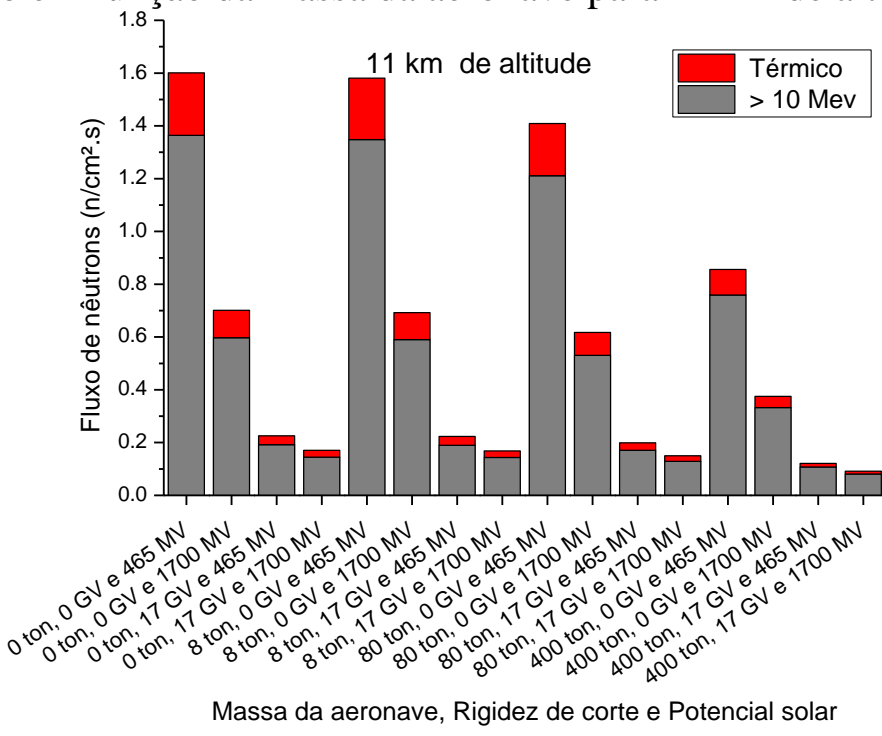

A Figura 5 apresenta a variação do fluxo de nêutrons rápidos acima de $10 \mathrm{MeV}$ (esquerda) e de nêutrons térmicos (direita) calculado pelo código EXPACS para altitude de $18000 \mathrm{~m}$ em função da massa da aeronave, nas situações de máximo e mínimo solar para rotas polares e equatoriais. 
Figura 5: Variação do fluxo de nêutrons rápidos (esquerda) e fluxo de nêutrons térmicos (direita) na posição do piloto em função da massa da aeronave para $18 \mathrm{~km}$ de altitude.

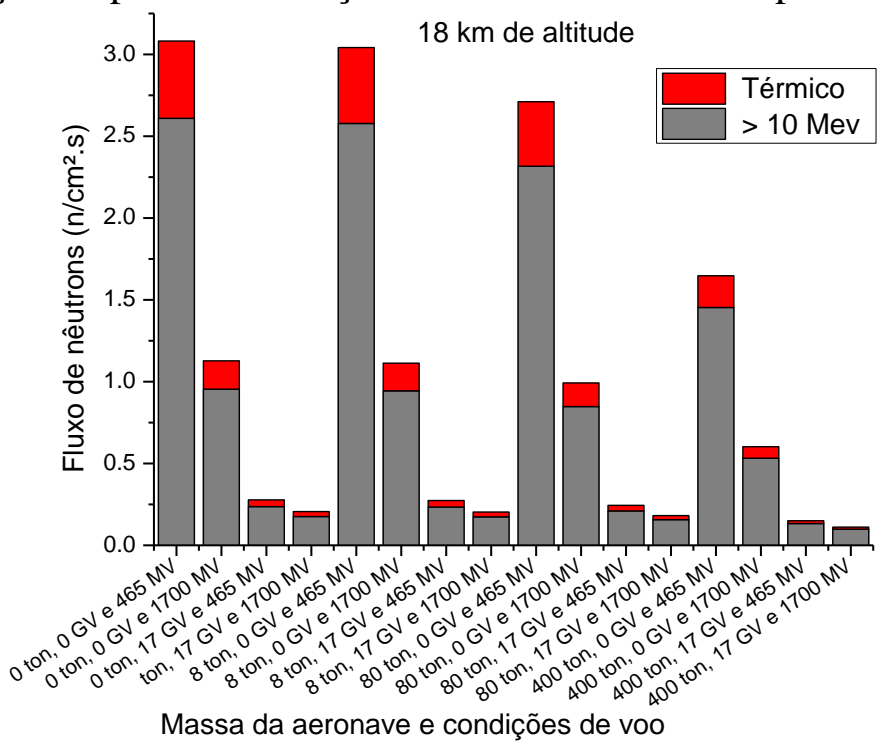

A Figura 6 apresenta a variação do fluxo de nêutrons acima de $10 \mathrm{MeV}$ e o fluxo de nêutrons térmicos com a massa total da aeronave para a pior situação ambiental - voos em rotas polares durante o período de mínimo solar - para duas altitudes: 11 e $18 \mathrm{~km}$, evidenciando que o fluxo de nêutrons é maior para maiores altitudes. Como a tendência de aeronaves é voar em maiores altitudes visando a economia de combustível, o problema dos efeitos da radiação em sistemas aviônicos é cada vez mais preponderante e deve-se buscar medidas mitigadoras contra estes efeitos a fim de garantir a segurança de voo. 
Figura 6: Variação do fluxo de nêutrons rápidos e térmicos em função da massa da aeronave para voos em rotas polares no período de mínimo solar para 11 e $18 \mathrm{~km}$ de altitude.

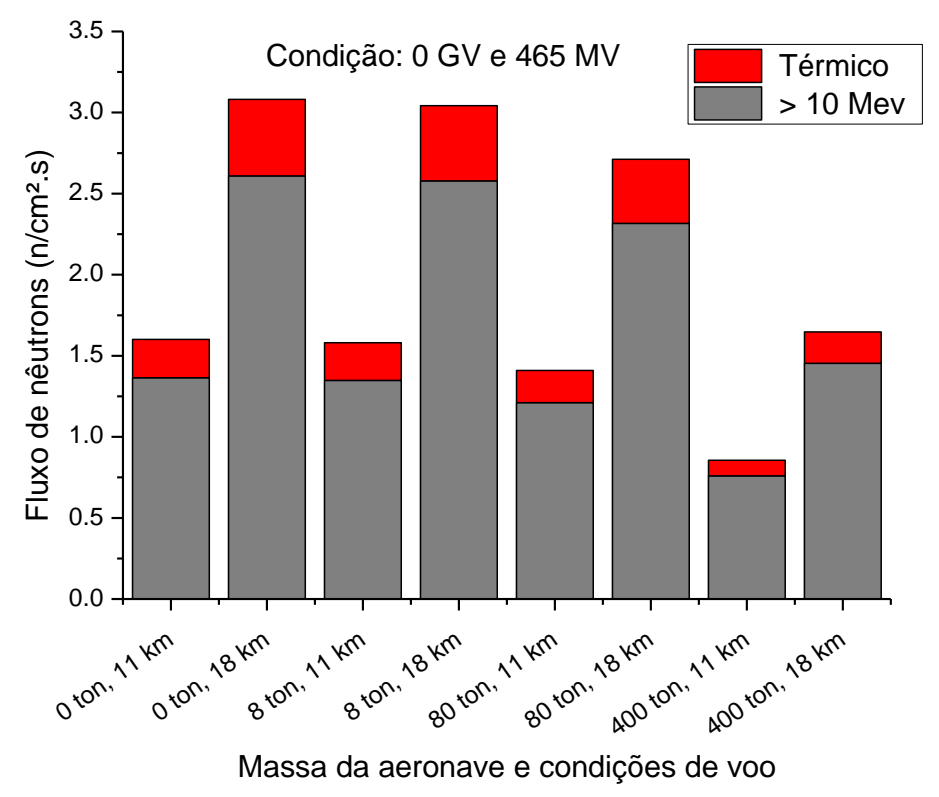

Utilizando os dados da seção de choque dos dispositivos D1 e D2 da tabela 1, os fluxos de nêutrons calculado pelo código EXPACS nas diferentes condições de voo para $11 \mathrm{~km}$ de altitude e as equações 3 e 4 é possível determinar a taxa de SEE esperada para cada condição de voo. A Figura 7 apresenta a taxa de falhas em função da massa da aeronave para estes dispositivos. 
Figura 7: Variação da taxa de falhas SEE em função da massa da aeronave e período solar para voos em $11 \mathrm{~km}$ de altitude

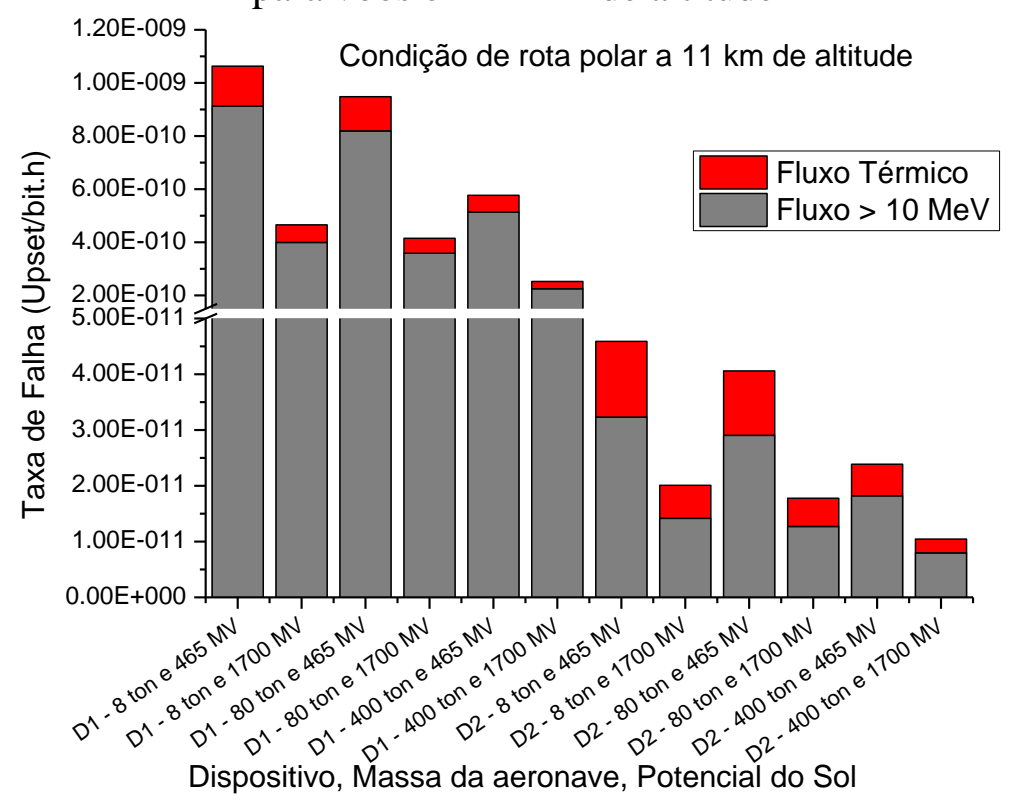

Condição de rota polar

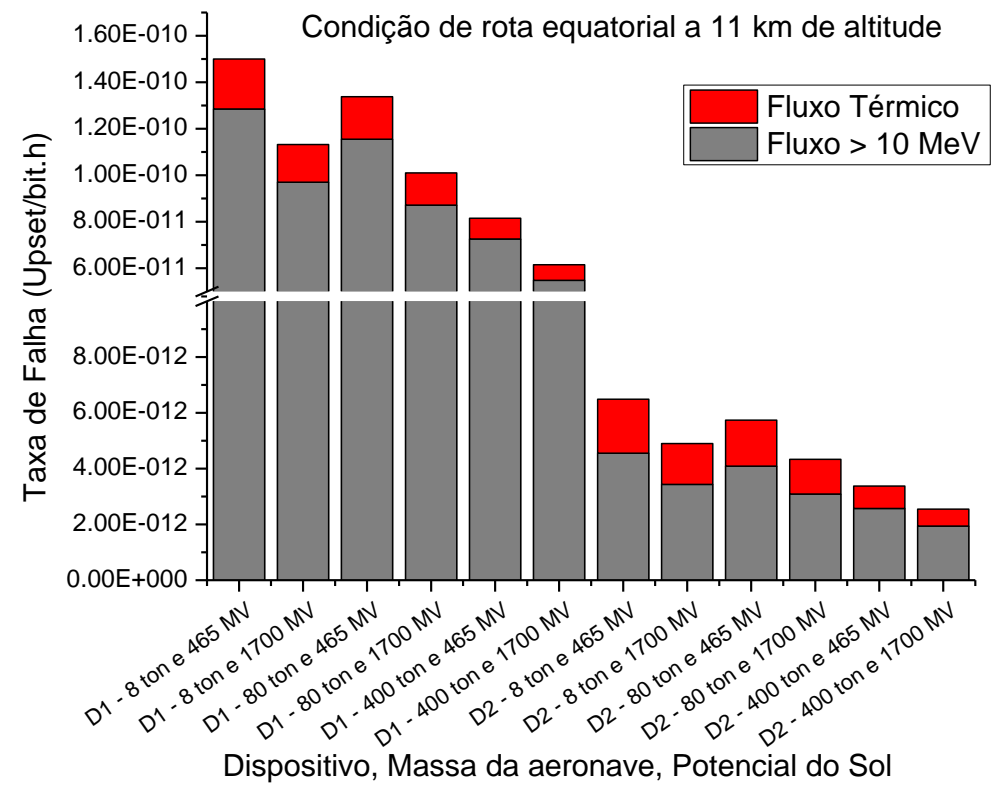

Condição de rota equatorial

A Figura 8 apresenta a taxa de falhas em função da massa da aeronave para estes mesmos dispositivos nas diferentes condições de voo para $18 \mathrm{~km}$ de altitude. 
Figura 8: Variação da taxa de falha SEE em função da massa da aeronave para voos em 18 km de altitude e em diferentes condições de rotas

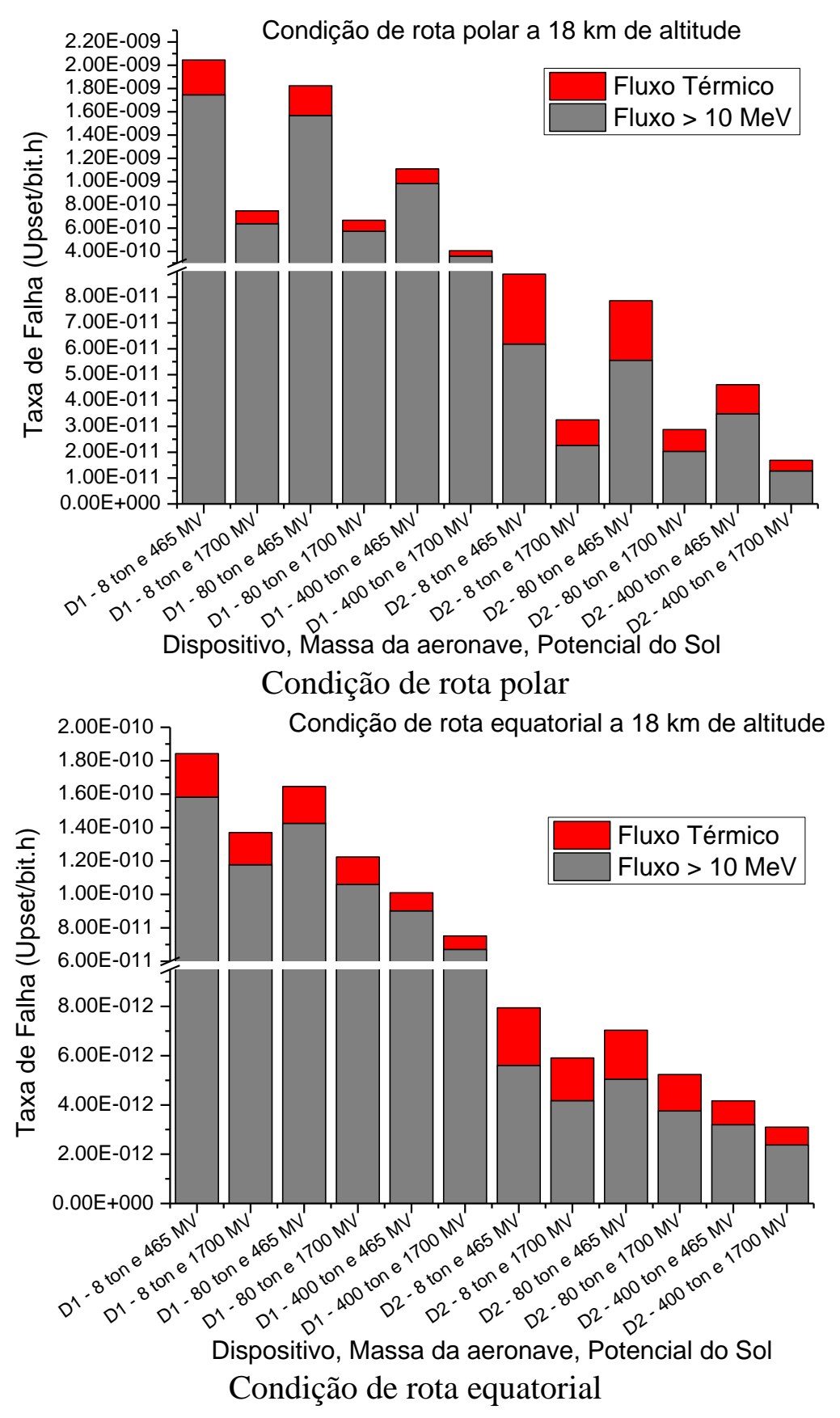

Através da Figura 7 pode-se perceber que as maiores taxas de falhas para voos em $11 \mathrm{~km} \mathrm{de}$ altitude ocorrem nas condições de rotas polares no período de mínimo e máximo solar respectivamente. 
O mesmo efeito é observado para altitudes de $18 \mathrm{~km}$ sendo condições de rotas polares e voos no período de mínimo e máximo solar as condições que apresentam maiores taxas de falhas a SEE. A Figura 9 apresenta as taxas de falha de cada dispositivo calculada para a pior condição de voo (rota polar, período de mínimo solar e aeronaves de pequeno porte), para 11 e $18 \mathrm{~km}$ de altitude.

Figura 9: Taxas de Falha SEE para dois dispositivos (D1 =1 e D2 = 2) típicos utilizados em aviônicos.

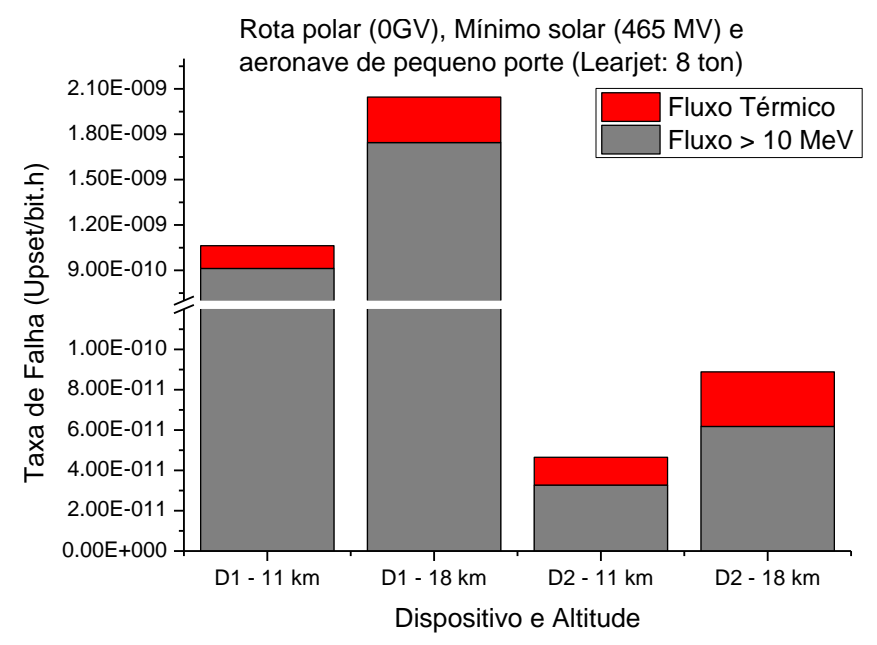

Para esta condição de voo o dispositivo D1 apresenta maiores taxas de falha tanto devida a nêutrons rápidos acima de $10 \mathrm{MeV}$ quanto a nêutrons térmicos. É importante ressaltar a influência da altitude para a taxa de falhas. Para o mesmo dispositivo a taxa de falhas quando operado a $18 \mathrm{~km}$ de altitude é maior do que quando operado a $11 \mathrm{~km}$ de altitude.

Como o dispositivo D1 é mais susceptível a falhas devido aos efeitos de SEE, todas as análises seguintes serão feitas para este dispositivo.

A Figura 10 apresenta a taxa de falhas do dispositivo devida a nêutrons com energia acima de $10 \mathrm{MeV}$ (esquerda) e a nêutrons térmicos (direita) a $11 \mathrm{~km}$ de altitude para as diferentes condições de voo. A Figura 11 apresenta a taxa de falhas do dispositivo devido a nêutrons com energia acima de $10 \mathrm{MeV}$ (esquerda) e para nêutrons térmicos (direita) a $18 \mathrm{~km}$ de altitude para as diferentes condições de voo. 
Figura 10: Taxa de falhas do dispositivo 1 em função da massa da aeronave devido a nêutrons com energia > $10 \mathrm{MeV}$ e para nêutrons térmicos a $11 \mathrm{~km}$ de altitude para as diferentes condições de voo

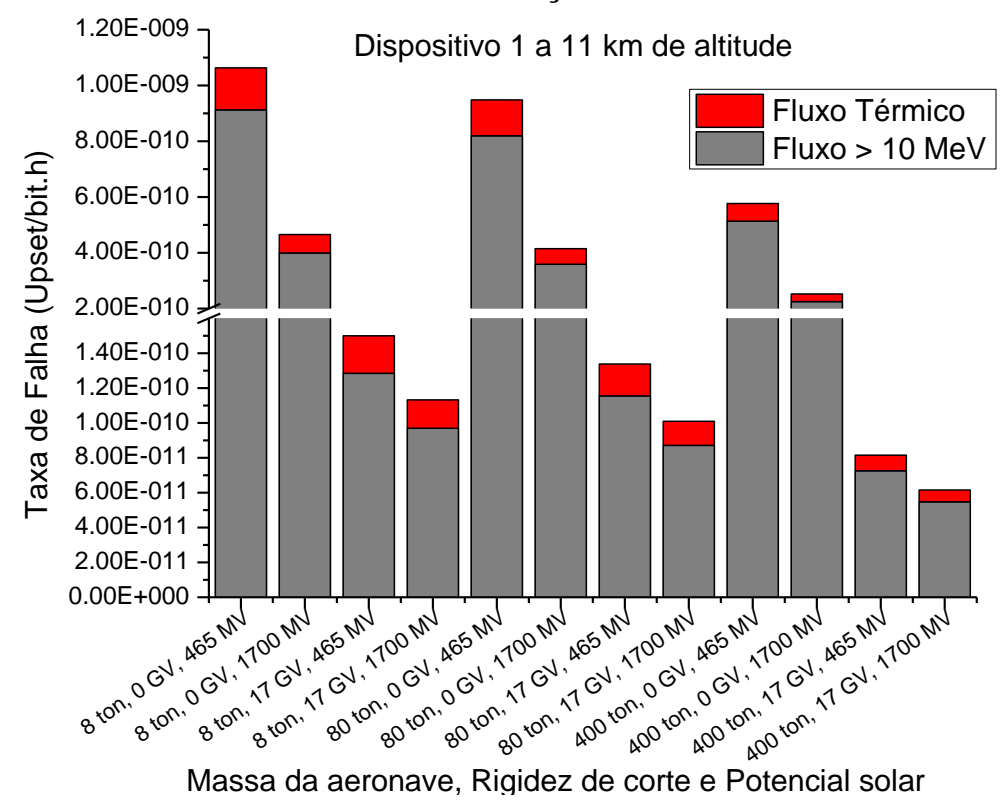

Figura 11: Taxa de falhas do dispositivo 1 em função da massa da aeronave devido a nêutrons com energia $>10 \mathrm{MeV}$ e para nêutrons térmicos a $18 \mathrm{~km}$ de altitude para as diferentes condições de voo.

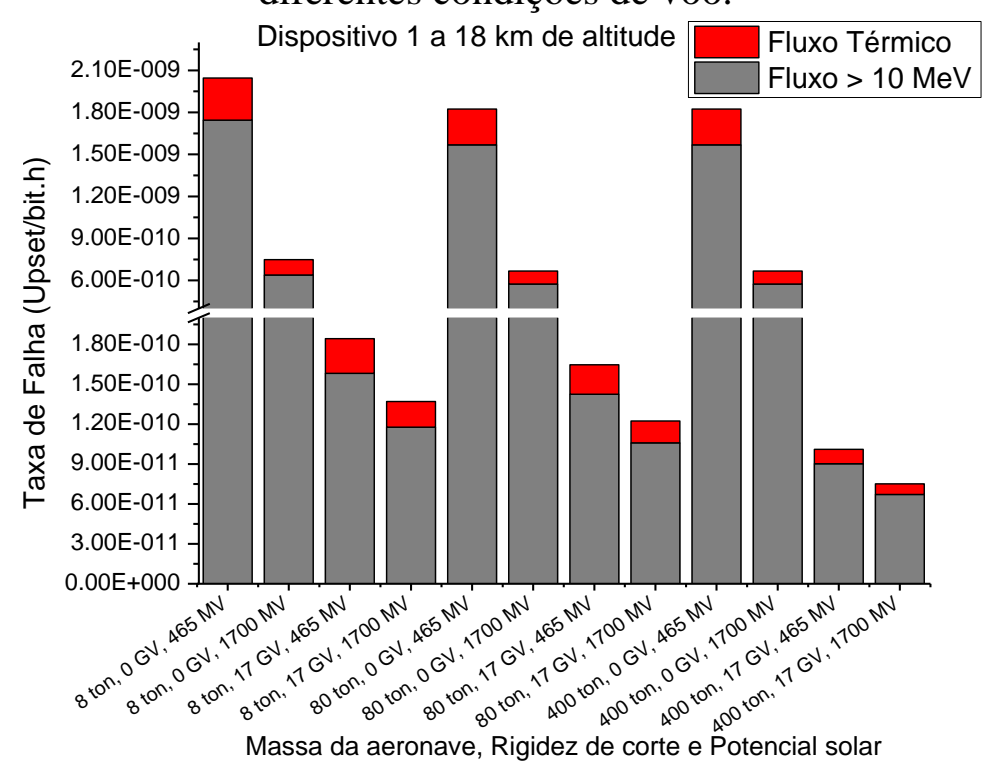

A partir das Figuras 10 e 11 que comparam a taxa de falhas em função da massa da aeronave fica evidente que aeronaves de menores massas apresentam maior taxa de falhas e que a pior situação 
de voo tanto para $11 \mathrm{~km}$ quanto para $18 \mathrm{~km}$ de altitude é referente à voos em rotas polares (0 GV) durante o mínimo solar (465 MV) enquanto que a situação que apresenta menor taxa de falhas para este dispositivo em questão é a condição de voos equatoriais (17 GV) durante o período de máximo solar (1700 MV).

A Figura 12 apresenta a diferença entre taxa de falhas do dispositivo D1 operando na condição de rota polar e mínimo solar e a taxa de falhas deste mesmo dispositivo operando na condição de rota equatorial e máximo solar para $11 \mathrm{~km}$ de altitude (esquerda) e $18 \mathrm{~km}$ de altitude (direita) em função da massa da aeronave.

Figura 12: Diferença entre a taxa de falhas SEE do dispositivo 1 para a condição de rota polar durante o mínimo solar e para a condição de rota equatorial durante o máximo solar em função da massa da aeronave e da altitude

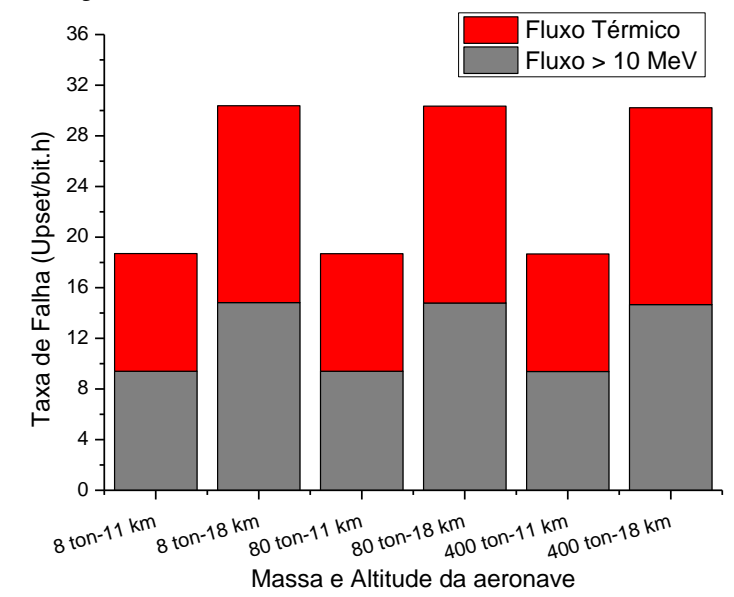

A taxa de falhas provocadas pelo fluxo de nêutrons rápidos acima de $10 \mathrm{MeV}$ na condição de voo em rota polar durante mínimo solar é aproximadamente 9,14 vezes maior que a taxa de falhas para rotas equatoriais durante o máximo solar para altitudes de $11 \mathrm{~km}$. Para a taxa de falhas devidas a nêutrons térmicos esta relação é de aproximadamente 9,28 vezes.

Já para voos em teto operacional de $18 \mathrm{~km}$ de altitude a taxa de falhas para o fluxo de nêutrons rápidos acima de $10 \mathrm{MeV}$ na condição de voo em rota polar durante mínimo solar é aproximadamente 14,8 vezes maior que a taxa de falhas para rotas equatoriais durante o máximo solar. Para a taxa de falhas devidas a nêutrons térmicos esta relação é de aproximadamente 15,6 vezes maior para condição de rota polar e período de mínimo solar. 
Supondo a utilização deste dispositivo no período de 100000 horas de voo é possível determinar a confiabilidade (C) deste dispositivo através da equação 5, a seguir:

$$
C(t)=e^{-R_{\text {total }} \cdot t}
$$

Onde o $\mathrm{R}_{\text {total }}$ é a soma das taxas de falhas devidas a SEE produzidos por nêutrons acima de $10 \mathrm{MeV}$ e nêutrons térmicos e t é o tempo de operação em horas.

A confiabilidade do dispositivo D1 em função do tempo de operação é apresentada na Figura 13 para $11 \mathrm{~km}$ de altitude nas condições de voo em rota polar durante o período de mínimo solar para aeronaves de pequeno, médio e grande porte.

Figura 13: Confiabilidade do dispositivo D1 em função do tempo. Condição de voo em rota polar durante o período de mínimo solar a $11 \mathrm{~km}$ de altitude para três diferentes categorias de aeronaves

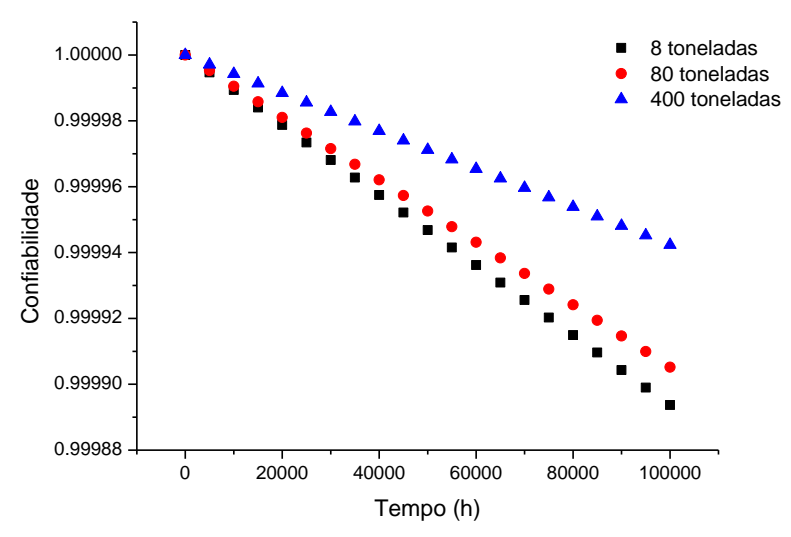

A Figura 14 apresenta a confiabilidade do dispositivo D1 para as mesmas condições descritas anteriormente com a aeronave voando a $18 \mathrm{~km}$ de altitude. 
Figura 14: Confiabilidade do dispositivo D1 em função do tempo. Condição de voo em rota polar durante o período de mínimo solar em $18 \mathrm{~km}$ de altitude para três diferentes categorias de aeronaves

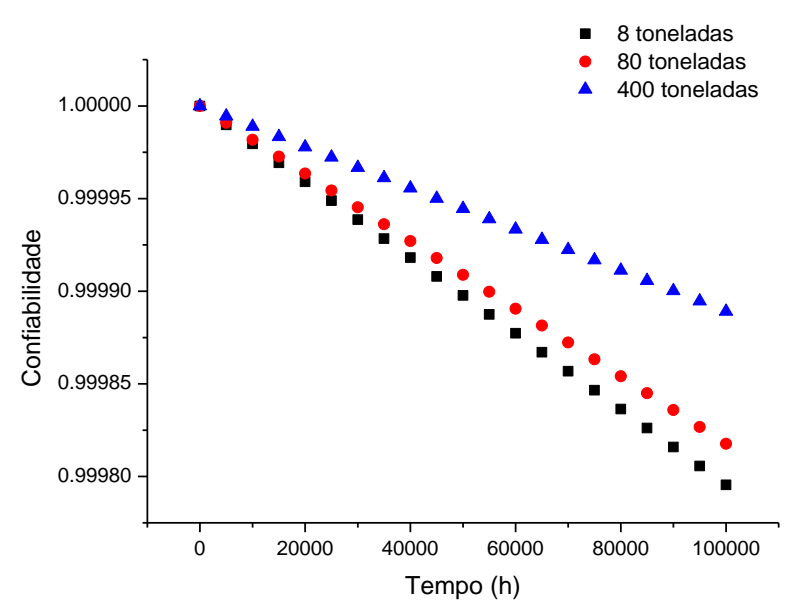

As Figuras 13 e 14 deixam evidente a influência da massa da aeronave na confiabilidade do dispositivo. Tanto para voos com teto operacional de $11 \mathrm{~km}$ quanto para operação em $18 \mathrm{~km}$ aeronaves de pequeno porte possuem menor confiabilidade quando comparada com aeronaves de maiores categorias.

\section{CONCLUSÕES}

Conforme mostrado neste trabalho os efeitos da radiação ionizante em dispositivos aviônicos também devem ser considerados na análise de segurança de voo, uma vez que falhas ocasionadas por estes mecanismos podem comprometer severamente a segurança de voo. Este é um assunto emergente, cuja importância tende a crescer com o passar do tempo devida ao avanço tecnológico dos dispositivos eletrônicos embarcados e a tendência de aumento do teto operacional de aeronaves comerciais e militares. Dessa forma evidencia-se que o mesmo precisa ser considerado nas análises de segurança de vôo em projetos envolvendo novas tecnologias da microeletrônica. Além disso, dada à diversidade de funções desempenhadas por dispositivos com alto grau de integração estudos e experimentos nesta área se mostram de significativa necessidade para o setor aeronáutico. 
Este trabalho apresenta um estudo de caso para dois dispositivos do tipo memória SRAM utilizadas em aviônicos, avaliando possíveis cenários hipotéticos de utilização e identificando por meio dos resultados da simulação computacional do fluxo de nêutrons atmosféricos com o EXPACS o ambiente mais critico que o dispositivo pode ser utilizado em sua operação normal, ou seja, voos em rotas polares durante o período de mínimo solar.

$O$ peso total da aeronave também exerce efeito decrescente na taxa de SEE por meio da influência da massa no fluxo de nêutrons, sendo que, para o mesmo dispositivo, as maiores taxas de falhas devidas a SEE ocorrem em aeronaves de pequeno porte. A taxa de falhas dos dispositivos utilizados em aeronaves de pequeno porte é aproximadamente $84 \%$ maior que a taxa de falhas dos dispositivos utilizados em aeronaves de grande porte e estas diferenças usualmente não são consideradas nas análises de segurança em projetos de aviônicos. Ressalta-se ainda o fato de que as aeronaves de pequeno porte do tipo "jato executivo" são projetadas e usualmente operadas em tetos de altitude superiores aos voos comerciais, o que pode ampliar ainda mais esta diferença nas taxas de falhas previstas e, portanto, implicar na diminuição da função confiabilidade dos dispositivos empregados nestas aeronaves.

Os resultados demonstram diferenças de até 15 vezes nas taxas de falha esperadas entre situações de voos polares de alta altitude no período de mínimo solar em relação aos voos equatoriais no período de máximo solar, resultantes das diferentes taxas de fluência de nêutrons térmicos e de alta energia para cada situação, podendo implicar em diferenças na função confiabilidade destes dispositivos.

\section{AGRADECIMENTO}

À CAPES (Programa Pró-estratégia) pela bolsa de mestrado do primeiro autor, ao ITA e ao IEAv pela oportunidade de estudar e agregar conhecimento. 


\section{REFERÊNCIAS}

FEDERICO, C. A. Dosimetria da radiação cósmica no interior de aeronaves no espaço aéreo brasileiro. 2011. 172f. Tese (Doutorado em Ciências na Área de Tecnologia Nuclear) - Instituto de Pesquisas Energéticas e Nucleares, São Paulo.

DYER, C. S. et al. Solar Particle Enhancements of Single-Event Effect Rates at Aircraft Altitudes. IEEE Transactions on Nuclear Science, v. 50, n. 6, p. 2038-2045, 2003.

MENDONÇA, R. R. S. de, Influência das variabilidades solar, geomagnética e atmosférica na modulação da intensidade de raios cósmicos. 2011. 94 f. Dissertação (Mestrado em Geofísica Espacial / Ciências do Ambiente Solar-Terrestre) - Instituto Nacional de Pesquisas Espaciais, São José dos Campos.

IEC - International Electrotechnical Commission. Process management for avionics Atmospheric radiation effects - Part 1: Accommodation of atmospheric radiation effects via single event effect within avionics electronic equipment. TECHNICAL SPECIFICATION IEC/TS 62396-1, .Suíça: IEC, 2006. 64p.

ATSB, Australian Transport Safety Bureau. Aviation Occurrence Investigation. AO-2008-070, Austrália: ATSB, 2011, 292p.

ICRU - International Commission on Radiation Units and Measurements. Fundamental quantities and units for ionizing radiation. ICRU Report 60, Bethesda: ICRU, 1998. 24p.

COOPER, N. G. National Security Science Los Alamos National Laboratory: The Invisible Neutron Threat. Disponível em: <http://lanl.gov/science/NSS/issue1_2012/story4full.shtml>. Acesso em: 15 dez. 2012.

O. L. Gonçalez. et al, Qualification of electronic components with respect to the cosmic radiation tolerance for space applications. Em: $4^{\mathbf{0}}$ Workshop sobre os efeitos da radiação ionizante em 
componentes eletrônicos e fotônicos de uso aeroespacial, 2012, São José dos Campos. Anais Werice Aeroespacial. v.1, pp. 51-56.

SATO T. e NIITA, K. Analytical Functions to Predict Cosmic-Ray Neutron Spectra in the Atmosphere, Radiation Research, v. 166, p. 544-555, 2006.

SATO T. et al. Development of PARMA: PHITS'-based Analytical Radiation Model in the Atmosphere. Radiation Research, v. 170, p. 244-259. 2008.

LEI, F. et al. An Atmospheric Radiation Model Based on Response Matrices Generated by Detailed Monte Carlo Simulations of Cosmic Ray Interactions. IEEE Transactions on Nuclear Science, v. 51, n. 6, p. 3442-3451, 2004.

LEI, F. et al. Improvement to and Validations of the QinetiQ Atmospheric Radiation Model (QARM). IEEE Transactions on Nuclear Science, v. 53, n. 4, p. 1851-1858, 2006.

IEC - International Electrotechnical Commission. Process management for avionics Atmospheric radiation effects - Part 5: Guidelines for assessing thermal neutron fluxes and effects in avionics systems. TECHNICAL SPECIFICATION IEC/TS 62396-5, .Suíça: IEC, 2008. 22p. 